\title{
Terminal Branch of Recurrent Human Laryngeal Nerve
}

\author{
Andréa Aparecida Ferreira Pascoal, ${ }^{1}$ Juliana Ruiz Fernandes, ${ }^{2}$ Cristiane Regina Ruiz, ${ }^{2}$ \\ Osmar Clayton Person, ${ }^{2}$ and Sergio Ricardo Rios Nascimento ${ }^{2}$
}

${ }^{1}$ EPM-UNIFESP, 04023-062 São Paulo, SP, Brazil

${ }^{2}$ Centro Universitário São Camilo, Avenida Nazaré, No. 1501, 04263-200 São Paulo, SP, Brazil

Correspondence should be addressed to Cristiane Regina Ruiz; crisrruiz@ig.com.br

Received 7 April 2014; Revised 24 August 2014; Accepted 29 August 2014; Published 16 September 2014

Academic Editor: Fabian Mohamed

Copyright (C) 2014 Andréa Aparecida Ferreira Pascoal et al. This is an open access article distributed under the Creative Commons Attribution License, which permits unrestricted use, distribution, and reproduction in any medium, provided the original work is properly cited.

\begin{abstract}
The importance of the recurrent laryngeal nerve in surgery on the anterior region of the neck has motivated many published papers on critical points of its pathway, relationship with the inferior thyroid artery, penetration in the larynx, division outside the larynx, and branches communicating with the internal branch of the superior laryngeal nerve. We analyze the terminal branches of the recurrent laryngeal nerve and their distribution through the laryngeal muscles. 44 laryngeal nerves had been dissected. Most frequently, the recurrent laryngeal nerve presents a division below or at the level of the lower margin of the cricoid cartilage (outside the larynx). One of these branches forms the communication with the internal branch of the superior laryngeal nerve, and the other penetrates the laryngeal space. Above the lower margin of the cricoid cartilage, the inferior laryngeal nerve issues a variable number of branches to muscles ( 3 to 7 ): to the posterior cricoarytenoid muscle; to the oblique and transversal arytenoid muscles; and to the lateral cricoarytenoid muscle and the thyroarytenoid muscle.
\end{abstract}

\section{Introduction}

The anatomy of the recurrent laryngeal nerve is important in medical practice applications $[1]$ and surgery $[2,3]$. Nerve injuries may occur in interventions performed on the anterior cervical region $[4,5]$, particularly during thyroidectomy $[6-$ 12] or parathyroidectomy [13-15]. Such injuries result in speech, breathing, and swallowing disorders [16], causing health and behavioral disorders.

Because of its variability, it is increasingly important knowing the anatomy of the recurrent laryngeal nerve [14, 1719]. One controversial matter hotly debated by scientists and anatomists [3] concerns the levels at which nerve divisions occur [20-27], along with the nature $[10,19,28-30]$ and destination of such branches $[22,31,32]$. Clarification of such issues is essential, since the clinical picture varies according to the injured branch [33].

Despite awareness of the importance of studying this nerve, anatomists and surgeons confront with controversies and doubts $[17,30,34-36]$ in the literature available. Better understanding of the anatomy of the recurrent laryngeal nerve and its terminal branches would also help fit the basis for establishing techniques for reinnervation of the larynx following recurrent laryngeal nerve injury $[4,37,38]$.

The objective of the research is to analyze the terminal branches of the recurrent laryngeal nerve and its distribution among the muscles of the larynx.

Our hypothesis is as recurrent laryngeal nerve has great clinical importance in general, however, there are many controversies in the literature on its terminal branches; it is expected that a more detailed description of these branches can answer these questions and assist in clinical medicine.

\section{Material and Methods}

Our sample was part of the Anatomy Laboratory of the Federal University of São Paulo and was composed of twentytwo larynges from adult individuals of both sexes; all these specimens had been fixed in $10 \%$ formalin. Their excision had included the pharynx, tongue, hyoid bone, infrahyoid muscles, and upper parts of the esophagus and the trachea. 
Both sides of the nerves were dissected, thus totaling 44 recurrent laryngeal nerves for analysis.

Firstly, the posterior pharyngeal/esophageal wall was dissected in order to make it possible to expose the anterior wall and reach the recurrent laryngeal nerve in the opening between the esophagus and the trachea. Using a magnifying glass, which increased $3 x$ the size of the structure, the nerve was dissected lengthwise and the branches were identified and followed until their terminations inside the muscles and the mucosa. After dissection, measurements were made with a digital meter in order to establish the relationship between the extralaryngeal division and the lower margin of the cricoid cartilage and between the intralaryngeal branches and the lateral margin of the cricoid cartilage, which was classified as upper, middle, and lower thirds for this purpose.

The chi-square test was used in the statistical analysis, with the purpose of correlating the variables, being the significant value of $P<0.05$.

\section{Results}

3.1. Division of the Recurrent Laryngeal Nerve below or at the Level of the Lower Margin of the Cricoid Cartilage (Extralaryngeal Division). In 31 cases (70.5\%), the recurrent laryngeal nerve bifurcated below (75\%) or at the level of the lower margin (25\%) of the cricoid cartilage, thus giving rise to the inferior laryngeal nerve and the branch communicating with the internal branch of the superior laryngeal nerve. The locations of the branches below the margin were between 1 and $10 \mathrm{~mm}(48 \%)$ or between 11 and $20 \mathrm{~mm}$ (43\%) from the lower margin of the cricoid cartilage. Considering this reference point for repair as the boundary between the recurrent laryngeal nerve and its inferior laryngeal branch, the inferior laryngeal nerve was the extension of the recurrent laryngeal nerve in the other 13 cases (29.5\%). No significant differences in lateral characteristics were found.

3.2. Number of Branches of the Inferior Laryngeal Nerve above the Lower Margin of the Cricoid Cartilage (Intralaryngeal). In the area of the larynx, the inferior laryngeal nerve was found to issue between three and seven branches and most often issued four to five branches (75\%). The branch that communicates with the internal branch of the superior laryngeal nerve was among these branches when the origin of the superior laryngeal nerve was above the lower margin of the cricoid cartilage.

3.3. Branches to the Posterior Cricoarytenoid Muscle (Figure 1). The posterior cricoarytenoid muscle was directly innervated by the inferior laryngeal nerve and was found to receive one $(52.3 \%)$ or two (36.4\%) branches emerging from the trunk at the lateral margin of the cricoid cartilage. Although we found that one branch tended to be located on the right side, the statistical calculation showed no significant difference. Dividing the lateral margin of the cricoid cartilage into thirds, we noticed that the origin of these branches to the muscle was in the lower third (44.1\%) or the middle third (49.2\%). In three cases $(6.8 \%)$, the presence of a branch shared by

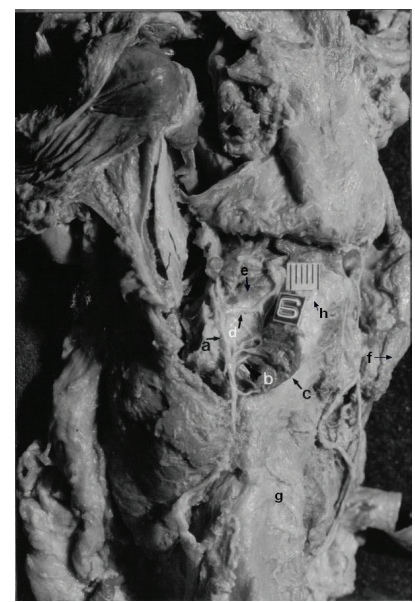

Figure 1: (a) Inferior laryngeal nerve; (b) (large arrow) a branch of inferior laryngeal nerve to posterior cricoarytenoid muscle; (c) posterior cricoarytenoid muscle; (d) a branch to arytenoid muscle (oblique and transversal) and to laryngeal mucose.

the posterior cricoarytenoid and oblique and transversal arytenoid muscles was noticed.

3.4. Branches to the Oblique and Transversal Arytenoid Muscles. The oblique and transversal arytenoid muscles were directly innervated by the inferior laryngeal nerve and received a single branch (90.7\%) or two branches (9.3\%). After detaching from the recurrent laryngeal nerve, they passed between the cricoid cartilage lamina and the posterior cricoarytenoid muscle. Using the same criteria used to locate the origin of the inferior laryngeal nerve, we found that the origin of this branch is from the upper third $(42.5 \%)$ or the middle third $(44.7 \%)$ of the lateral margin of the cricoid cartilage. After penetrating the muscle, the branch divided into two (45.6\%) or three (39.1) smaller branches.

3.5. Branches to the Lateral Cricoarytenoid and Thyroarytenoid Muscles (Figure 2). More often (86.4\%), the lateral cricoarytenoid and thyroarytenoid muscles received a single branch, while less often they received two branches emerging from the inferior laryngeal nerve, in the upper third (50.0\%) or the middle third (40.9\%) of the lateral margin of the cricoid cartilage. After penetrating the thyroid arytenoid muscle, the branch that went to this muscle issued two (36.0\%), three (26.0\%), or several (26.0\%) small branches.

\section{Discussion}

4.1. Division of the Recurrent Laryngeal Nerve below or at the Level of the Lower Margin of the Cricoid Cartilage (Extralaryngeal Division). Division of the recurrent laryngeal nerve into two or three branches before penetrating the area of the larynx was already known and its occurrence has varied between different studies. The frequency of division absence within the lower margin has been reported to be $78 \%$ [20], most 


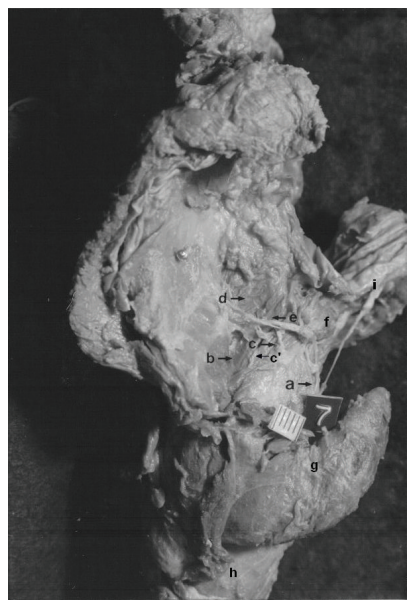

Figure 2: (a) Inferior laryngeal nerve; (b) lateral cricoarytenoid muscle; (c) a branch of inferior laryngeal nerve to the lateral cricoarytenoid muscle; (d) thyroarytenoid muscle; (e) terminal portion of the inferior laryngeal nerve to the thyroarytenoid muscle.

cases [8], 30\% [33], 73\% [21], 100\% [3, 23, 28, 30], 70\% [17], $15 \%[22,29], 43 \%$ [34], usually [8], 41.2\% [18], 65\% [14], 58\% [10], 87\% [27], and 65\% [12, 14].

In order to explain vocal fold paralysis relating to abduction or adduction, some authors $[8,20,33]$ considered the nerve branches to be motor branches for abduction or adduction or mixed branches [17]. It is now known that they are sensitive and motor branches [3, 22-24, 26, 28].

This division has been reported in the literature: in Nguyen et al. [27], with division occurring up to $1 \mathrm{~cm}$ from the margin (54\%), between 1 and $2 \mathrm{~cm}$ from it (42.5\%), or more than $2 \mathrm{~cm}$ from it (7\%); in Salama, up to $2 \mathrm{~cm}(96 \%)$; in Nemiroff and Katz [18], up to $1.7 \mathrm{~cm}(43.5 \%)$ or from 1.8 to $2.3 \mathrm{~cm}(38,1 \%)$; in Katz [10], between 0.6 and $4 \mathrm{~cm}$; in Williams [28], up to $2.5 \mathrm{~cm}$; and in Steinberg et al. [30], mostly at $2 \mathrm{~cm}$. We found that the division occurred between 0.01 and $0.1 \mathrm{~cm} \mathrm{(48 \% )} \mathrm{or} \mathrm{between} 0.11$ and $0.20 \mathrm{~cm} \mathrm{(43 \% )} \mathrm{from} \mathrm{the}$ lower margin of the cricoid cartilage in our material.

4.2. Branches to the Posterior Cricoarytenoid Muscle. Nguyen et al. [27] classified the innervation of the posterior cricoarytenoid muscle into three types. In type I, occurring in $66.6 \%$, the muscle received one branch that could be single (53.4\%), be bifurcated (6.6\%), or be trifurcated (6.6\%) and its origin is in the lower three-quarters of the inferior horn of the thyroid cartilage. In type II, occurring in $26.7 \%$, the muscle received two branches, and the superior nerve and the branch could join together to the arytenoid muscles (6.7\%). In type III, in $6.7 \%$, there were three single branches $(5 \%)$ or one branch joined to the branch to the arytenoid muscles (1.7\%), our results thus confirming the results obtained by Nguyen et al. The number of branches has varied between different studies. Mu et al. [32] and Freche and Boudin [19] reported one branch, Laux and Guerrier [35] found one branch that could be double, Sanders reported one or two branches, with one of them joined to the branch to the arytenoid muscles,
Hiroto et al. [24] found two to three branches, and Winckler [31] mentioned two branches.

The origin of these branches was in the lower third (44.1\%) or the middle third (49.2\%) of the lateral margin of the cricoid cartilage. Eller et al. [39] found an average of three to four branches to the muscle (range from one to eight): two or three directly from the anterior division of the nerve and one (range from one to three) joined to the branch to the arytenoid muscles. They used the lower margin of the cricoarytenoid junction as a reference point to state that the origin of the first branch was between 3.4 and $4.2 \mathrm{~mm}$ above this point and that the last branch originated around $9.5 \mathrm{~mm}$ above this point. In our study, we adopted a division of the lateral margin of the cricoid cartilage into thirds (upper, middle, and lower) as the reference point for branch emission, in order to avoid measurement errors during dissection.

4.3. Branches to the Oblique and Transversal Arytenoid Muscles. The authors, Laux and Guerrier [35], Hiroto et al. [24], $\mathrm{Mu}$ et al. [32], Sanders et al. [13], Winckler [31], Rueger [26], and Nguyen et al. [27], agreed that the arytenoid muscles received a single branch that detached from the upper inferior laryngeal nerve (near the cricoarytenoid junction) and that inside the muscle the branch admitted in various ways. However, according to Freche and Boudin [19], the branch to the arytenoid muscles is very inconstant, and Nordland [6] even stated that it was absent. Maranillo et al. [38] found four to eight branches (average of 5.5) to these muscles, but only two branches (one on each side) originated from the upper recurrent laryngeal nerve, and the others originated from the internal branch of the superior laryngeal nerve. In $88 \%$, the branch to the muscles was connected to the branch to the posterior cricoarytenoid muscle and the origin occurred behind the cricoarytenoid junction (97\%).

In our study, the oblique and transversal arytenoid muscles received a single branch (90.7\%) or two branches (9.3\%) that moved forward the posterior cricoarytenoid muscle. These branches originated from two levels: the upper third $(42.5 \%)$ or the middle third $(44.7 \%)$ of the lateral margin of the cricoid cartilage, taking the reference point of the lower margin of the cricoid cartilage.

4.4. Branches to the Lateral Cricoarytenoid and Thyroarytenoid Muscles. In descriptions of the lateral cricoarytenoid and thyroarytenoid muscles innervation, Laux and Guerrier [35], Winckler [31], Freche and Boudin [19], Sanders et al. [13], Keros and Nemanic [3], Hiroto et al. [24], and Nguyen et al. [27] agreed that the trunk of the inferior laryngeal nerve moved forward to form a curve towards these muscles. At this level, it could penetrate one of these muscles and issue branches to both muscles, or alternatively, divide into two branches such that each one would go to one of the muscles. The branch to the thyroarytenoid muscle ended in a sort of fan of small branches. In our study, we found similar innervation and noticed that this change of direction by the branch (forwards) occurred in either upper third (42.5\%) or middle third $(44.7 \%)$ of the lateral margin of the cricoid cartilage. With the aim of resecting the nerve in cases of 
adductor spasmodic dysphonia, Scheid et al. [1] found that the branch to the muscle was located approximately $4.23 \mathrm{~mm}$ above the inferior tubercle in the inferior margin of the thyroid cartilage and that, in $87.5 \%$ of the cases, the division was into many branches. We found that this division was into two (36\%), three (26\%), or several (26\%) small branches. Maranillo et al. [38] mentioned that the muscles could also be innervated by the internal branch of the superior laryngeal nerve. Such occurrences were not confirmed in the present study, although in $18.3 \%$ of the cases we noticed communication between the two nerves laterally to these muscles, such that the muscle branches came out from this point. The participation of the internal branch was found to be proprioceptive, rather than motor. According to Maranillo et al. [38], in $43.3 \%$ of the cases there were three branches to the lateral cricoarytenoid muscle, which we believe to be divisions of the branch originating from the recurrent laryngeal nerve. According to these authors, the two branches were joined to both muscles in only $10 \%$ of the cases, whereas we noticed this occurrence in $86.4 \%$, in the present study.

\section{Conclusions}

In conclusion, very often, the recurrent laryngeal nerve is divided below or at the level of the lower margin of the cricoid cartilage. Above the lower margin of the cricoid cartilage, the inferior laryngeal nerve can issue a varying number of branches (3 to 7). The inferior laryngeal nerve issues one or two branches to the posterior cricoarytenoid muscle, always in the areas of the larynx and in the lower and middle thirds of the lateral margin of the cricoid cartilage. The inferior laryngeal nerve issues one branch to the oblique and transversal arytenoid muscles, always in the area of the larynx and in the middle and upper thirds of the lateral margin of the cricoid cartilage. The inferior laryngeal nerve issues a single branch to the lateral cricoarytenoid and thyroarytenoid muscles, always in the area of the larynx and in the middle and upper levels of the lateral margin of the cricoid cartilage. Thus we can conclude that the anatomy of the recurrent laryngeal nerve is quite variable, but the descriptions in this work are consistent with studies in the literature, thereby assisting in the medical clinic.

\section{Conflict of Interests}

The authors declare that there is no conflict of interests regarding the publication of this paper.

\section{Acknowledgments}

This research was designed from the scientific curiosity of a great teacher of anatomy who lived to stimulate the minds of their disciples with new questions that subsequently generated product as a scientific paper. The authors appreciate the presence of Professor Dr. Nader Wafae in our lives, a brilliant man who unfortunately was unable to witness the completion of this work.

\section{References}

[1] S. C. Scheid, D. P. Nadeau, O. Friedman, and R. T. Sataloff, "Anatomy of the thyroarytenoid branch of the recurrent laryngeal nerve," Journal of Voice, vol. 18, no. 3, pp. 279-284, 2004.

[2] E. F. Ziegelman, "Laryngeal nerves: surgical importance in relation to the thyroid arteries, thyroid gland and larynx," Archives of Otolaryngology, vol. 18, no. 6, pp. 793-808, 1933.

[3] P. Keros and D. Nemanic, "The terminal branching of the recurrent laryngeal nerve," Practica Oto_Rhino_Laryngologica, vol. 29, no. 1, pp. 5-10, 1967.

[4] P. J. Doyle, R. E. Brummett, and E. C. Everts, "Results of surgical section and repair of the recurrent laryngeal nerve.," Laryngoscope, vol. 77, no. 8, pp. 1245-1254, 1967.

[5] D. C. Green and P. H. Ward, "The management of the divided recurrent laryngeal nerve," Laryngoscope, vol. 100, no. 7, pp. 779-782, 1990.

[6] M. Nordland, "The larynx as related to surgery of the thyroid. Based on an anatomical study," Surgery, Gynecology \& Obstetrics, vol. 51, pp. 449-459, 1930.

[7] D. D. Berlin, "The recurrent laryngeal nerves in total ablation of the normal thyroid gland," Surgery, Gynecology and Obstetrics, vol. 60, pp. 19-26, 1935.

[8] F. H. Lahey, "Exposure of the recurrent laryngeal nerves in thyroid operations," Surgery, Gynecology \& Obstetrics, vol. 78, pp. 239-244, 1944.

[9] J. E. Skandalakis, C. Droulias, N. Harlaftis, S. Tzinas, S. W. Gray, and J. T. Akin Jr., "The recurrent laryngeal nerve," The American Surgeon, vol. 42, no. 9, pp. 629-634, 1976.

[10] A. D. Katz, "Extralaryngeal division of the recurrent laryngeal nerve. Report on 400 patients and the 721 nerves measured," The American Journal of Surgery, vol. 152, no. 4, pp. 407-410, 1986.

[11] N. Wafae, M. C. Vieira, and A. Vorobieff, "The recurrent laryngeal nerve in relation to the inferior constrictor muscle of the pharynx," Laryngoscope, vol. 101, no. 10, pp. 1091-1093, 1991.

[12] A. B. Salama and P. McGrath, "Recurrent laryngeal nerve and the posterior fascial attachment of the thyroid gland," Australian and New Zealand Journal of Surgery, vol. 62, no. 6, pp. 444-449, 1992.

[13] G. Sanders, R. Y. Uyeda, and M. S. Karlan, "Nonrecurrent inferior laryngeal nerves and their association with a recurrent branch," The American Journal of Surgery, vol. 146, no. 4, pp. 501-503, 1983.

[14] M. S. Karlan, B. Catz, D. Dunkelman, R. Y. Uyeda, and S. Gleischman, "A safe technique for thyroidectomy with complete nerve dissection and parathyroid preservation," Head and Neck Surgery, vol. 6, no. 6, pp. 1014-1019, 1984.

[15] J. Gavilan and C. Gavilan, "Recurrent laryngeal nerve. Identification during thyroid and parathyroid surgery," Archives of Otolaryngology-Head and Neck Surgery, vol. 112, no. 12, pp. 1286-1288, 1986.

[16] R. D. Henderson, A. Boszko, and A. W. P. VanNostrand, "Pharyngoesophageal dysphagia and recurrent laryngeal nerve palsy," The Journal of Thoracic and Cardiovascular Surgery, vol. 68 , no. 4, pp. 507-512, 1974.

[17] S. Sunderland and W. E. Swaney, "The intraneural topography of the recurrent laryngeal nerve in man," The Anatomical Record, vol. 114, no. 3, pp. 411-426, 1952.

[18] P. M. Nemiroff and A. D. Katz, "Extralaryngeal divisions of the recurrent laryngeal nerve. Surgical and clinical significance," American Journal of Surgery, vol. 144, no. 4, pp. 466-469, 1982. 
[19] C. Freche and G. Boudin, "Anatomy and physiology of recurrent laryngeal nerve," Journal Francais d'Oto-Rhino-Laryngologie, vol. 31, no. 1, pp. 51-64, 1982.

[20] C. Weeks and J. W. Hinton, "Extralaryngeal division of the recurrent laryngeal nerve. Its significance en vocal cord paralysis," Annals of Surgery, vol. 116, pp. 251-258, 1942.

[21] W. G. Armstrong and J. W. Hinton, "Multiple divisions of the recurrent laryngeal nerve," Archives of Surgery, vol. 62, pp. 532539, 1951.

[22] P. Kluyskens, "Sur la localisation de la division du nerf laryngé inferieur," Comptes Rendus de l'Association des Anatomistes, vol. 39, pp. 36-41, 1952.

[23] H. Pichler and A. Gisel, "The clinical significance of the ramification of the recurrent laryngeal nerves; a critical anatomical study," The Laryngoscope, vol. 67, no. 2, pp. 105-117, 1957.

[24] I. Hiroto, Y. Toyozumi, and Y. Yatake, "Comparative anatomy of the laryngeal nerves of mammals," Journal of Otolaryngology of Japan, vol. 71, no. 2, pp. 212-216, 1968.

[25] R. C. Kratz, “The identification and protection of the laryngeal motor nerves during thyroid and laryngeal surgery: a new microsurgical technique.," Laryngoscope, vol. 83, no. 1, pp. 5978, 1973.

[26] R. S. Rueger, "The superior laryngeal nerve and the interarytenoid muscle in humans: an anatomical study," Laryngoscope, vol. 82, no. 11, pp. 2008-2031, 1972.

[27] M. Nguyen, C. Junien-Lavillauroy, and C. Faure, "Anatomical intra-laryngeal anterior branch study of the recurrent (inferior) laryngeal nerve," Surgical and Radiologic Anatomy, vol. 11, no. 2, pp. 123-127, 1989.

[28] A. F. Williams, "The nerve supply of the laryngeal muscles," The Journal of Laryngology and Otology, vol. 65, no. 5, pp. 343-348, 1951.

[29] S. Schneider, "L'anatomie du nerf recurrent dans la loge thyroidienne; ses variations et leurs incidences sur la chirurgie du corps thyroïde," Helvetica Chirurgica Acta, vol. 6, pp. 482-486, 1956.

[30] J. L. Steinberg, G. J. Khane, C. M. C. Fernandes, and J. P. Nel, "Anatomy of the recurrent laryngeal nerve: a redescription," Journal of Laryngology and Otology, vol. 100, no. 8, pp. 919-927, 1986.

[31] G. Winckler, "Remarques sur linnervation motrice et sensitive des muscles du larynx," Comptes Rendus de L'Association des Anatomistes, vol. 35, pp. 424-428, 1948.

[32] L. Mu, I. Sanders, B.-L. Wu, and H. F. Biller, “The intramuscular innervation of the human interarytenoid muscle," Laryngoscope, vol. 104, no. 1, pp. 33-39, 1994.

[33] B. T. King and R. L. Gregg, "An anatomical reason for the various behaviors of paralysed vocal cords," Annals of Otology, Rhinology \& Laryngology, vol. 57, pp. 925-944, 1948.

[34] W. H. Rustad, "Revised anatomy of the recurrent laryngeal nerves: surgical importance, based on the dissection of 100 cadavers," The Journal of Clinical Endocrinology \& Metabolism, vol. 14, no. 1, pp. 87-98, 1954.

[35] G. Laux and Y. Guerrier, "Linnervation des muscles du larynx," Comptes Rendus de $l$ Association des Anatomistes, vol. 46, pp. 46-62, 1959.

[36] I. Sanders, B.-L. Wu, L. Mu, Y. Li, and H. F. Biller, "The innervation of the human larynx," Archives of OtolaryngologyHead and Neck Surgery, vol. 119, no. 9, pp. 934-939, 1993.

[37] R. L. Crumley, "Update: ansa cervicalis to recurrent laryngeal nerve anastomosis for unilateral laryngeal paralysis," Laryngoscope, vol. 101, no. 4 I, pp. 384-388, 1991.
[38] E. Maranillo, X. Leon, C. Orus, M. Quer, and J. R. Sanudo, "Variability in nerve patterns of the adductor muscle group supplied by the recurrent laryngeal nerve," Laryngoscope, vol. 115, no. 2, pp. 358-362, 2005.

[39] R. L. Eller, M. Miller, J. Weinstein, and R. T. Sataloff, "The innervation of the posterior cricoarytenoid muscle: exploring clinical possibilities," Journal of Voice, vol. 23, no. 2, pp. 229-234, 2009. 

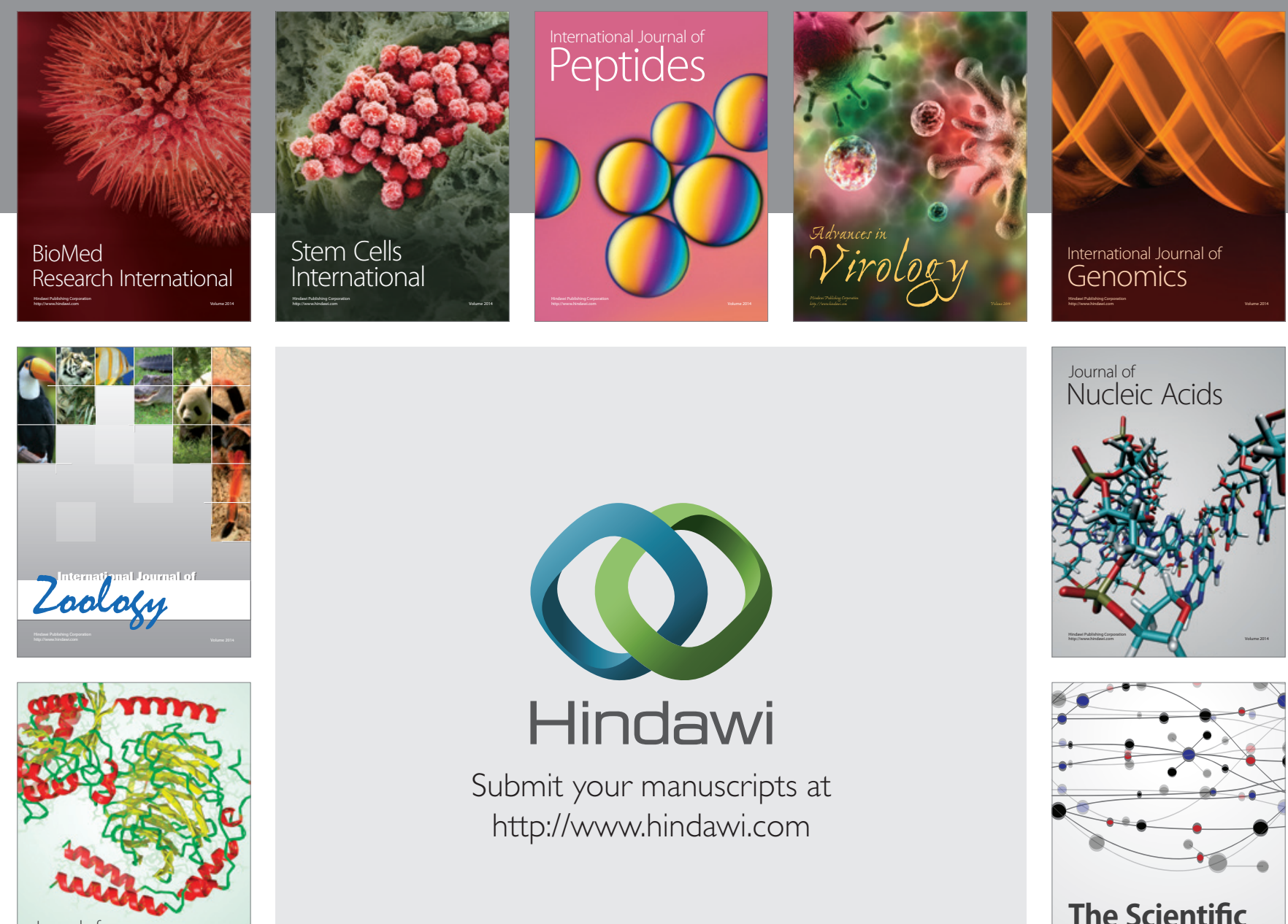

Submit your manuscripts at

http://www.hindawi.com

Journal of
Signal Transduction
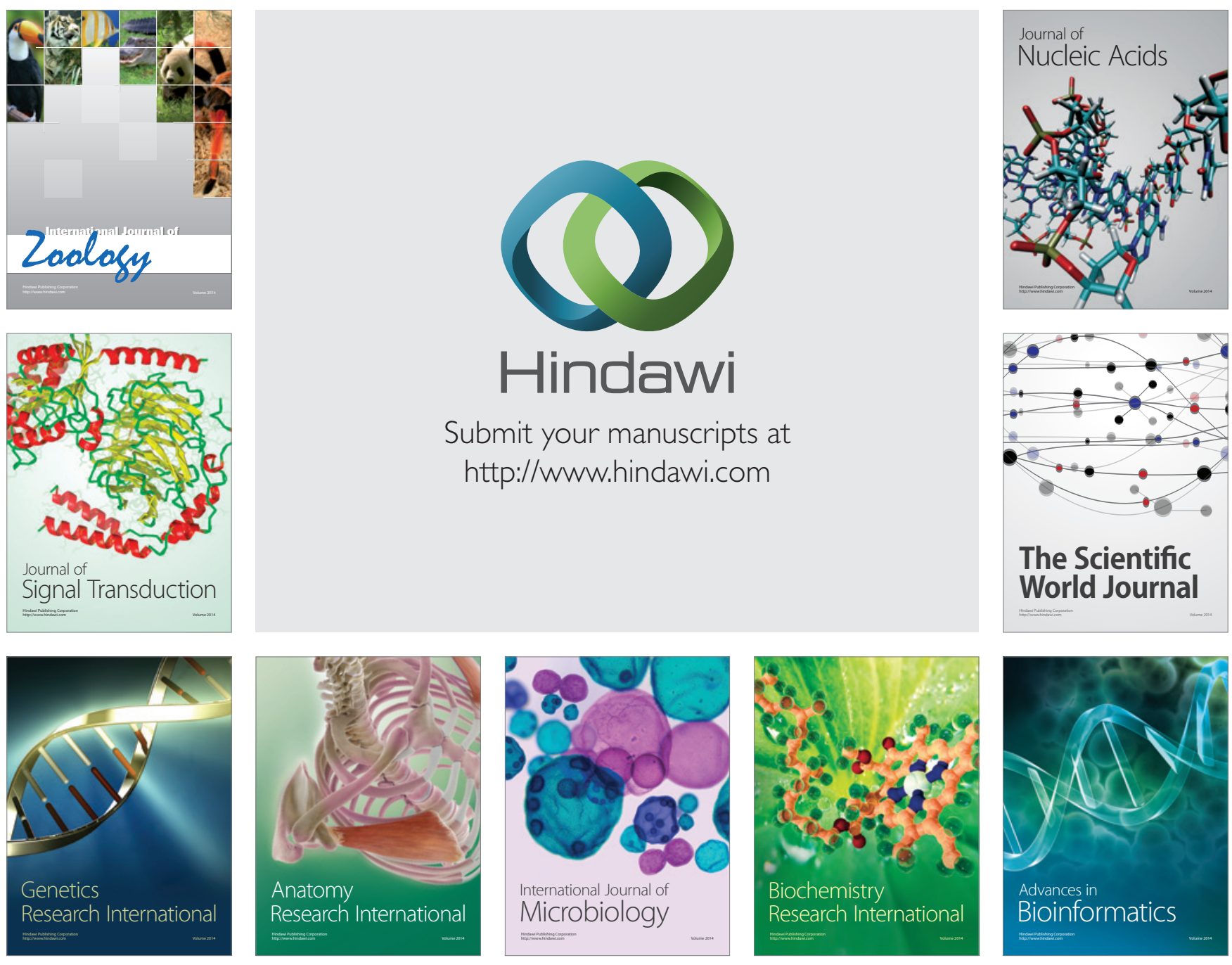

The Scientific World Journal
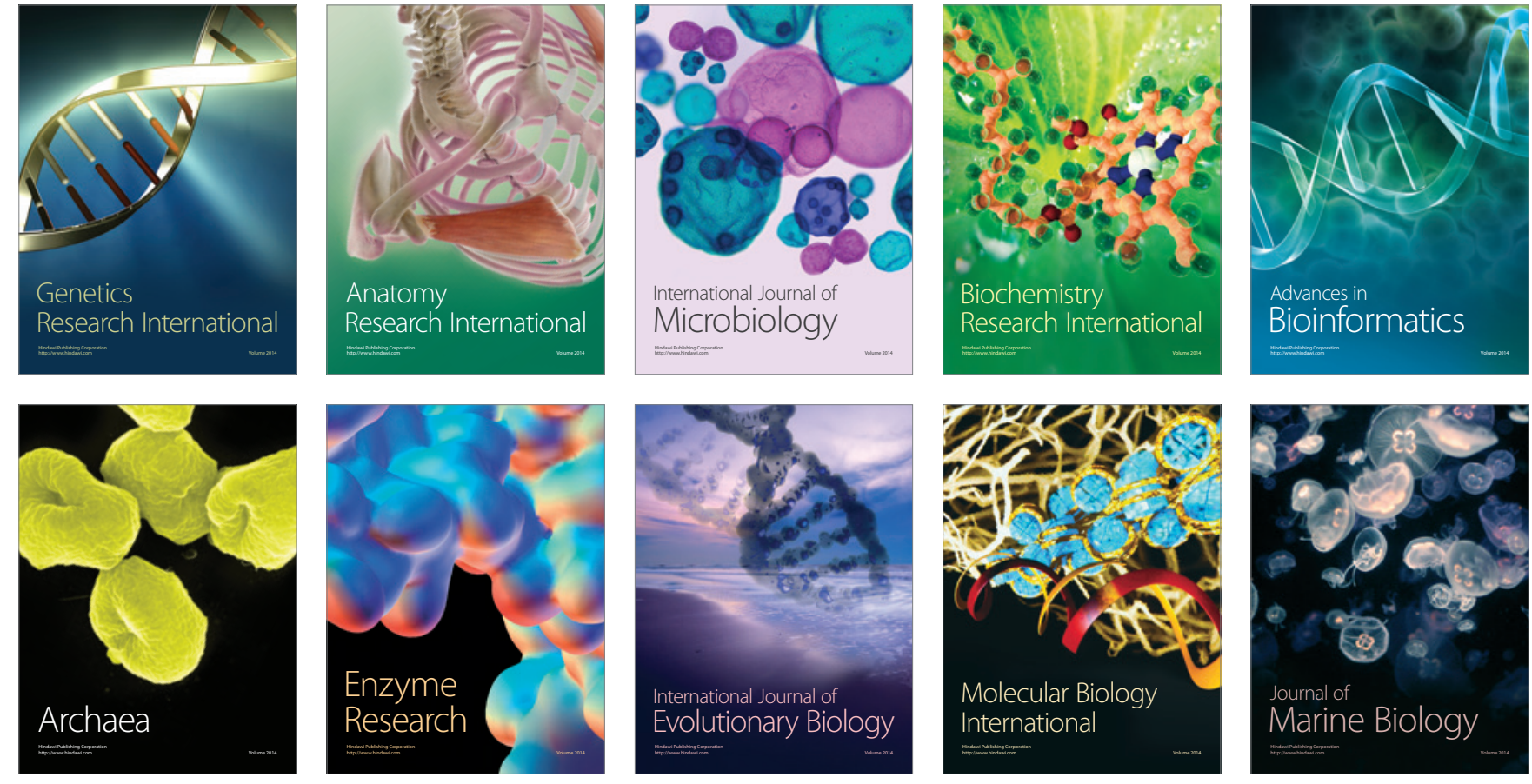